\title{
Three-dimensional Müller-Lyer illusion
}

\author{
ROMI NIJHAWAN \\ University of California, Berkeley, California
}

\begin{abstract}
Three-dimensional (3-D) variants of the Müller-Lyer pattern were created to address the question of where along the path of information flow in the visual system the illusion might occur. These variants, which yielded a robust illusion, included dihedral angles in place of the arrowheads of the classical pattern. The enormous difference in the shape of the resulting retinal image, compared with that of the classical pattern, makes it difficult to explain the present illusion by resorting to image-processing theories such as selective filtering (Ginsburg, 1984, 1986) or depth processing (Gregory, 1963, 1966, 1968). It was also shown that this 3-D illusion is homologous with the classical illusion, and that the two may thus share a common causal mechanism. A new type of 3-D figure, which yielded the same retinal image as did the classical pattern, was then employed. However, since the figure was 3-D, its shape in spatial coordinates was very different compared to that of the classical pattern. The magnitude of the illusion obtained with this figure was half that of the classical pattern. This finding suggests that the illusion might be caused by processes that occur after the computation of depth. All three experiments indicated that the illusion may be produced later in the processing stream than has previously been suggested.
\end{abstract}

Geometrical illusions, which usually involve judgments of size, curvature, angular extent, and so forth, were among the first topics addressed by experimental psychologists. However, despite the fact that the experimental procedures for investigating geometrical illusions are rather straightforward, a rigorous theory that adequately explains even one of the illusions has yet to be proposed. Consequently, interest in the study of geometrical illusions has waxed and waned. The Müller-Lyer is the most extensively investigated geometrical illusion for which a satisfactory theory still eludes psychologists.

The pattern originally presented by Müller-Lyer (1889) himself consists of a "longer half" (in which the horizontal line appears longer) and a "shorter half" (in which the horizontal line appears shorter) (Figure 1). Each half of the illusion pattern consists of two parts: the inducing elements (IEs) and the test element (shaft). The shafts extending between the IEs, although they have the same physical length, appear to the average observer to be unequal by more than $20 \%$.

Numerous variations of the original pattern have been created for the study of the phenomenon in its full generality, the simplest of which was the removal of the shafts (see Figure 2a, after Brentano, 1892). In other important variations, the IEs have been presented in the shape of

This paper is based in part on a dissertation submitted by the author to Rutgers University, New Brunswick. The author wishes to acknowledge the contribution of Shinsuke Shimojo, who provided ideas for new experiments. The author also wishes to thank Irvin Rock and Beena Khurana for many helpful suggestions, and to thank an anonymous reviewer for comments on an earlier draft. Correspondence may be addressed to Romi Nijhawan, Department of Psychology, Tolman Hall, University of California, Berkeley, CA 94720.

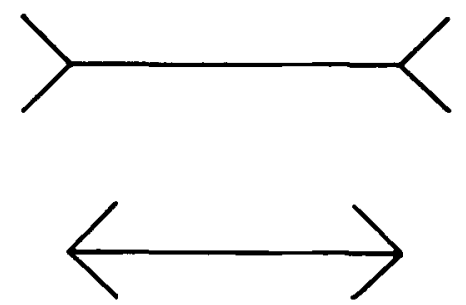

Figure 1. The original Müller-Lyer pattern (Müller-Lyer, 1889).

semicircles (Figure 2b, after Delboeuf, 1892) and brackets (Figure 2c, after Brentano, 1892). All of these patterns yield the illusion originally observed, although its strength is reduced. A rigorous theory must be general enough to explain the illusion found in all these variations, as well as the others that exist too. Unfortunately, in most of the explanations forwarded (see, e.g., Ginsburg, 1984, 1986; Gregory, 1963, 1966, 1968), researchers have sought primarily to explain the illusion in the classical figure and have generally not given the variants due consideration.

The Müller-Lyer illusion is usually presented as a line drawing on a two-dimensional (2-D) surface in the frontal plane. Therefore, the shape of the pattern and the shape of the projected retinal image are more or less identical. A major consequence has been that most of the proposed explanations hinge on the shape of the retinal image of the figure (see, e.g., Ginsburg, 1984, 1986; Gregory, 1963, 1966, 1968). The present study was designed to show that the illusion occurs readily with 3-D patterns. This not only challenges the traditional method of presentation of the figure, but also shows that the shape of the retinal image may not be critical to the understanding of the illusion, as has also been suggested by previ- 
The dihedral angles were positioned relative to the shafts so that in one half of the pattern, the concave sides faced away from the shaft (longer half), and in the other half, the concave sides faced toward the shaft (shorter half).

\section{Method}

Subjects. Thirty subjects from the Rutgers University subject pool took part in the experiment for course credit. All were naive with respect to the purpose of the experiment.

Apparatus. The apparatus consisted of a rectangular frame that held the 3-D Müller-Lyer pattern. The longer half of the pattern was placed on top of the frame and the shorter half on the bottom. A track was constructed on the bottom part of the frame; on it, a cart could be moved smoothly to the left or the right, with the pull of one of two strings. The cart carried one of the four dihedral angles, while the other three dihedral angles were fixed. The displacement of the cart caused the distance between the bottom pair of angles to increase or decrease and also caused the covering/ uncovering of a line, so that its length varied. All four dihedral angles were constructed from transparent Plexiglas.

Stimuli. Two illusion patterns were created. The planes of each dihedral angle intersected at $90^{\circ}$. For one of the patterns, thin strips of fluorescent material were used to outline each rectangular plane (Figure 3); for the other, random dots were placed on the interior of the planes, thus removing all contours from the dihedral angles (Figure 4). The outlines shown in Figure 4 are meant only for the purpose of illustration. A string covered with fluorescent material extending between the midpoints of the "spines" of dihedral angles represented the shaft. With the exception of the four dihedral angles (visible as outlines for one pattern and as random dots for the other) and two horizontal lines (shafts), the room was completely dark. Black lights were used as illumination. The length of the top shaft was fixed at $45.7 \mathrm{~cm}$. Each rectangle of the dihedral angles measured $15.2 \times 11.4 \mathrm{~cm}$. For the random-dot pattern, the radius of each dot was $.3 \mathrm{~cm}$ and 25 dots were used to define each rectangle. The left dihedral angles of the top and bottom pairs were offset so that the subjects could not simply use imaginary vertical lines to make the judgments. The distance between the subject's eyes and the middle of the display was $152.4 \mathrm{~cm}$.

Procedure. There were two groups of subjects. The first group consisted of 16 subjects who were shown the outline version of the pattern. The second group consisted of 14 subjects who were shown the random-dot version of the pattern. Each subject was given six trials, three ascending and three descending. The ascending and descending trials were given alternately, with half the subjects beginning with ascending trials and the other half with descending. The ascending trial began with the length of the comparison line set at $25.4 \mathrm{~cm}$; the descending trial, with the length set at $66 \mathrm{~cm}$. The subjects were asked to equate the lengths of the two lines by pulling on the appropriate string. On any given trial, the subject was asked to move the variable dihedral angle in only one direction, left or right. The settings were recorded from a scale fixed alongside the track, out of the subject's view. The subjects held their heads stationary while viewing the display binocularly.

\section{Results and Discussion}

Outline version group. For this group, the mean setting of the line length for the shorter half was $54 \mathrm{~cm}$. Since the top shaft (longer half) was $45.7 \mathrm{~cm}$ in length, the magnitude of the illusion was $8.3 \mathrm{~cm}$ or $18.2 \%[t(15)=12.11$, $p<.001]$.

The 3-D image shown in Figure 3 contains both 2-D and 3-D information. Is it possible that certain 2-D (e.g., see Figure 5) properties of the outline version of the pattern might have caused the illusion? The part of Figure 3
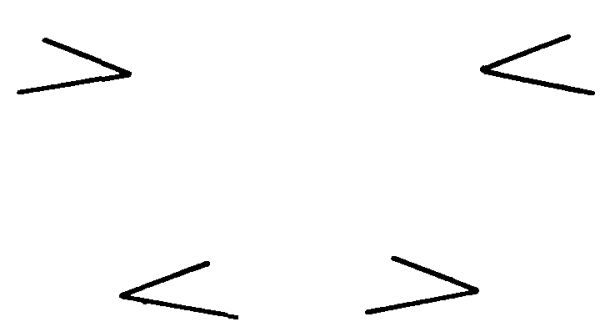

Figure 5. The top portion of the 3-D pattern (outlines) used in Experiment 1. This resembles the 2-D pattern shown in Figure 2a.

shown in Figure 5, which resembles the classical figure, might have been responsible for the illusion while the 3-D shape of the pattern had no effect. The outcome with the random-dot version, which does not contain the stimulus property shown in Figure 5, resolves this issue.

Random-dot version group. For this group, the mean setting of the shaft length for the longer half was $55 \mathrm{~cm}$, which is $9.3 \mathrm{~cm}$ longer than that of the shorter half. Thus, the shaft of the longer half appeared to be $20.4 \%$ longer than that of the shorter half $[t(13)=10.79, p<.001]$. A significant illusion was thus observed with the randomdot version, even though the lines that could have produced a 2-D illusion were removed.

Two main questions remained unaddressed following Experiment 1. Was it possible that in the random-dot version of the pattern, the illusion, even though not caused by the stimulus property shown in Figure 5 , might have been caused by some other information present in the retinal image that had nothing to do with the 3-D representation of the pattern? The image of the IE of the randomdot pattern, which is shaped like a "blob," contains information similar to that contained in the variant shown in Figure 2c, where the "blob" has the shape of a bracket. Could the mere placing of dot clusters at the shaft ends have produced the illusion?

The second question concerned whether it was appropriate to group the new 3-D illusion with the classical illusion. Might the 3-D illusion belong to a different category of phenomena? If the present illusion is indeed homologous with the Müller-Lyer illusion, then there ought to be some characteristics common to the two. Both these issues were addressed in Experiment 2.

\section{EXPERIMENT 2}

Experiment 2 was designed for the purpose of studying the variation in the illusion magnitude as a function of the angle between the planes of the dihedral angles. One characteristic of the classical pattern is that smaller angles tend to yield a stronger illusion than larger angles do (Lewis, 1909). (The angles referred to here are those between the two line segments of the IEs.) Lewis (1909), who used angles between $20^{\circ}$ and $180^{\circ}$, found that the maximum illusion occurred with a $20^{\circ}$ angle and that the illusion decreased as the angle was increased. If such a 
variation in magnitude were to be found with the 3-D pattern as well, then we could conclude that a common causal mechanism underlies the classical and the 3-D MüllerLyer illusions.

Experiment 2 consisted of five conditions, four of which involved binocular viewing of the pattern, with angles between the planes of the dihedral angles set at $30^{\circ}, 60^{\circ}$, $90^{\circ}$, and $120^{\circ}$. The fifth condition consisted of monocular viewing of the $30^{\circ}$ display. It was reasoned that monocular viewing would not yield the 3-D shape of the random-dot pattern, whereas binocular viewing would. A comparison of the two conditions of viewing would then allow an assessment of the role of the 3-D shape of the pattern in causing the illusion. In fact, one could conceive of the monocular condition as a $0^{\circ}$ condition. In this condition, motion parallax was eliminated by having the subject look through a small aperture, so that when the subject's head moved, the pattern would go out of view, thus ensuring head-stationary viewing. If the 3-D shape of the dihedral angles plays an important role in producing the illusion, the magnitude of the illusion found with binocular viewing should be greater than the magnitude found with monocular viewing.

\section{Method}

Subjects. Fifty subjects from the subject pool at the University of California at Berkeley participated in Experiment 2 for course credit. All subjects were naive with respect to the purpose of the experiment.

Apparatus. A new apparatus was constructed for Experiment 2. The planes of the dihedral angles were connected with hinges so that the angle between the planes could be varied. Moreover, instead of the two halves of the display being separated vertically, they were placed laterally to minimize the possibility that the subjects might use a strategy such as drawing imaginary lines to make their length judgments. Both the track for the motion of the cart and the covering/uncovering mechanism were similar to those used in Experiment 1.

Stimuli. So that the generality of the results could be studied, the stimuli were very different in size from the stimuli in the previous experiments. The shaft length of the standard display was $20.32 \mathrm{~cm}$. The lower and upper limits of length of the variable shaft were 11.43 and $29.21 \mathrm{~cm}$, respectively. Each rectangular plane of the dihedral angles was $5.08 \times 6.60 \mathrm{~cm}$. As in the second condition of Experiment 1, fluorescent random dots were placed on the interior of the planes, and the perimeter of the planes was invisible. The radius of each dot was $.1 \mathrm{~cm}$, and 20 dots were used to define each rectangle. In order to ensure that the 3-D nature of the display was not perceived with monocular viewing, the dots were placed so that a texture density gradient ought not to have been present in the retinal image.

Procedure. With the exception of variation of the angles, the binocular conditions were identical to those in Experiment 1. In the monocular condition, the subject wore plain glasses with one side occluded and looked with one eye through an opening that was $1 \times 1 \mathrm{~cm}$. When the subjects had finished making the length judgments, they were asked to give an estimate of the angle between the planes that they perceived by manually adjusting two planes under normal room lighting conditions.

\section{Results and Discussion}

Binocular viewing. As with the classical pattern, the illusion was strongest with the smallest angle and weakest with the largest angle. The magnitudes of the illusion at $30^{\circ}, 60^{\circ}, 90^{\circ}$, and $120^{\circ}$ were $22.23 \%, 21.49 \%, 17.78 \%$, and $13.69 \%$, respectively. An analysis of variance for independent samples yielded an $F(3,36)=7.41, p<.01$. In Figure 6, the magnitude of the illusion is plotted as a function of the angle between the planes.

The main outcome of the binocular conditions supports the view that the 3-D Müller-Lyer illusion being studied is indeed homologous with, rather than merely analogous to, the classical illusion.

Monocular viewing. In the monocular condition (using the $30^{\circ}$ pattern), the magnitude of the illusion was $8.82 \%$, which is two fifths of the magnitude obtained in the binocular condition. A $t$ test for independent samples comparing the $30^{\circ}$ monocular and binocular conditions yielded a $t(18)=25.71, p<.001$.

In the monocular condition, the two planes of the dihedral angles were collapsed into one, and the subjects perceived single 2-D surfaces on which the dots were located. This was evident when, following this condition, the subjects were asked to adjust the planes in accordance with the way in which they perceived the dotted planes; all the subjects collapsed the two planes into one. The difference between the magnitudes of the illusion in the monocular and the binocular conditions indicates that the 3-D representation of the pattern plays an important role in the production of the illusion.

As noted before, the illusion with the random-dot version (binocular viewing) might have been caused by the mere presence of dots at the shaft ends, which are shaped like "blobs." This illusion is similar to the bracket version shown in Figure 2c. The illusion magnitude of $8.82 \%$, in the monocular condition, may indeed have been due to this factor.

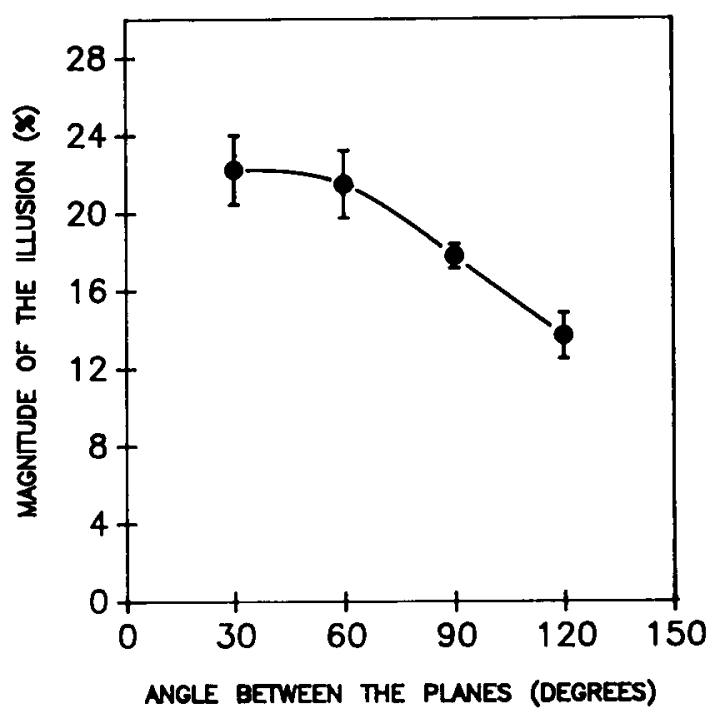

Figure 6. The graph in which the magnitude of the lllusion experienced by the subjects is plotted as a function of the angles between the planes of the dihedral angles (dotted planes). Each point represents a mean based on 10 scores. Error bars represent the standard error of the mean. 
Another observation from Experiment 2 is that in the angle adjustment task, following the binocular conditions, there was a consistent underestimation of the angle between the planes of the dihedral angles. Thus, the depth perception was not perfect, and the 3-D shape of the dihedral angles was not perceived veridically (see also the Results and Discussion section of Experiment 3).

\section{EXPERIMENT 3}

Experiment 3 also addressed the question of the level of processing that underlies the Müller-Lyer illusion, but with a rather different technique. When the figure is studied, the shaft and the IEs typically lie in the same plane-the plane of the surface on which the figure is drawn. In Experiment 3 , this coplanarity was removed by arranging the IEs in a plane that was at right angles to the shaft (as is depicted schematically in Figure 7). Nonetheless, the projection of this 3-D structure in the plane of the retina was identical to that of the classical 2-D figure (see Figure 7). The 3-D objects composed of cubes shown in Figure 7 are, however, not the stimuli used in the experiment, their purpose being only to illustrate the 3-D structure. The actual stimuli were constructed from thin fluorescent material.

The 3-D shapes were designed to address the following question: Is the shape of the 3-D display (as it is represented after 3-D shape processing), or the shape of the retinal image (projected by the display), more critical in causing the illusion? If what matter for the illusion are processes such as retinal neural interactions or selective spatial filtering, the illusion should be unaffected by the orthogonal orientation of the IEs relative to the shaft, because what is crucial for these processes is the information that they receive from the retinal image. On the other hand, if what matters is how the resulting structure is rep-
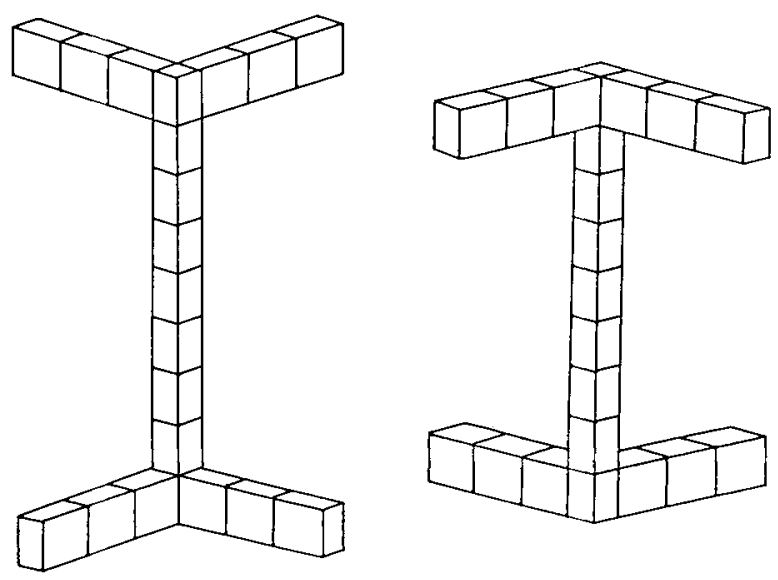

Figure 7. The cube figures show the 3-D shape of the stimuli used in Experiment 3. The actual stimuli with this 3-D structure, however, were made from thin fluorescent material. resented in 3-D space, the illusion might disappear. This is perhaps because in the 3-D display the concave (or convex) side of the IEs lies orthogonal to the shaft, whereas in the 2-D figure the concave side of the IEs faces either toward the shaft (for the shorter half) or away from the shaft (for the longer half). Another reason for why the illusion might disappear will be discussed in the General Discussion section. It was hypothesized that the illusion found with the present 3-D display would either be reduced in strength or disappear entirely.

\section{Method}

Subjects. Sixteen subjects from the Rutgers University community volunteered to take part in Experiment 3. All subjects were naive with respect to the purpose of the experiment.

Apparatus. A system of pulleys allowed a rectangular block of wood to slide vertically on a surface in the frontal plane. As before, the subject varied the length of one shaft to equate it to the other. For varying the length of the comparison shaft, a metallic object (counterweight), attached to the block of wood by two strings, could be moved up or down on another vertical surface near the subject. Thus, the variable part of the apparatus consisted of the following: a block of wood that could slide smoothly on a surface; a system of pulleys and two strings, with one end of each string connected to the block of wood; and a counterweight attached to the other ends of the strings.

When suspended freely, this system was in balance, owing to friction between the counterweight and its surface and between the wood block and its surface. A slight force upward or downward allowed the wood block to move smoothly. The wood block carried one IE and a mechanism, similar to the one used for the previous experiments, to cover or uncover the comparison line. The subject could move the counterweight up or down, and by doing so decrease or increase the length of the shaft extending between one pair of IEs. For measurements, a scale that was out of the subject's view was fixed next to the counterweight.

Stimuli. Two vertical lines made of fluorescent material were placed side by side. On the ends of each line, a pair of IEs, made of strips of fluorescent material, was attached. The IEs were all $90^{\circ}$ angles and the length of each angle arm was $3.2 \mathrm{~cm}$. The IEs were oriented so that an IE pair lay either in the same plane as the shaft (control) or in planes orthogonal to the plane of the shaft. The former will be referred to as the planes-parallel display and the latter as the planes-perpendicular display.

Traditionally, when the Müller-Lyer illusion is studied, the observer's line of sight meets the midpoint of the shafts at right angles. As can be seen from Figure 7, if this condition were met for the planes-perpendicular display, the angles would project straight lines on the retina. To allow the projected shape of the IEs to be identical to that of the classical pattern, the shafts of the planesperpendicular stimulus were placed below the observer's eye level. To match the viewing conditions, the classical (planes-parallel) display was also placed below the observer's eye level. For both displays, the observer's line of sight made an angle of $25^{\circ}$ at the midpoint of the shafts. The longer half, located toward the subject's left, contained the standard shaft $(31.1 \mathrm{~cm})$. The top IE of the right pair (shorter half) could be moved up or down with the mechanism described earlier, which also made the comparison shaft longer or shorter. All stimuli were viewed binocularly while the subjects held their heads still. The distance between the subject's eyes and the midpoint of the test lines was $101 \mathrm{~cm}$ (approximately).

Procedure. Experiment 3 consisted of two conditions: planesperpendicular and planes-parallel. Eight of the 16 subjects participated in the planes-parallel condition, which essentially involved 
the classical illusion, the only difference being that the subject's line of sight did not meet the midpoints of the shafts at right angles. This served as a control for the planes-perpendicular condition, in which the other 8 subjects participated.

Each subject was given three ascending and three descending trials, alternately. Half the subjects began with an ascending trial and the other half with a descending trial. For the ascending trial, the length of the comparison shaft was set at $18.4 \mathrm{~cm}$; for the descending trial, it was set at $43.8 \mathrm{~cm}$. For a given trial, the subject was instructed to move the counterweight only in one direction, up or down, to equate the shaft lengths.

\section{Results and Discussion}

The mean setting for the shaft of the shorter half for the planes-parallel group was $36.1 \mathrm{~cm}$. Thus, on the average this shaft was set $5.0 \mathrm{~cm}$ longer than the shaft of the longer half (an illusion of magnitude of $16.07 \%$ ). This illusion is somewhat weaker than the illusion typically observed, which may be due to the observer's line of sight not meeting the midpoint of the shaft at right angles.

The mean setting of the shaft for the shorter half (retinally defined) of the planes-perpendicular group was $33.6 \mathrm{~cm}$. Thus, for this display, the mean setting for this shaft was $2.5 \mathrm{~cm}$ longer than the shaft of the longer half (retinally defined). The parenthetic qualifiers used above are necessary, because the given descriptions for the planes-perpendicular display may not be valid if we consider the 3-D shape of the pattern. The above setting amounts to an illusion magnitude of $8.04 \%$. In short, as we go from the planes-parallel display to the planesperpendicular display, the magnitude of the illusion is halved. The two groups differ significantly in the magnitude of illusion perceived. A $t$ test for independent samples yielded a $t(14)=6.78, p<.001$.

The retinal images of the two halves of the planesperpendicular display are identical to those of the planesparallel display. If all that mattered in the production of the illusion was a peripheral process associated with the retinal neural interaction or 2-D selective spatial filtering, then the illusion should be unaffected by a change in the orientation of the IEs relative to the shafts. Thus, the outcome of Experiment 3 is inconsistent with the retinal neural interaction and selective filtering theories of the Müller-Lyer illusion. The outcome of Experiment 3 supports the hypothesis that the illusion is affected by the representation of the extent between the IEs in 3-D space.

The observation that the illusion did not totally vanish may at first seem surprising. However, it was observed during the experiment that subjects never perceived the IEs to be oriented at right angles to the shaft. This observation is quite similar to the underestimation of the dihedral angles obtained in Experiment 2 (see the Results and Discussion section of Experiment 2). The perceived angle was somewhere between the objective angle $\left(90^{\circ}\right)$ and the angle in the 2-D image $\left(0^{\circ}\right)$. Observations that the computation of depth of a 3-D shape is influenced by the retinal image features abound in the literature (see, e.g., Gogel, 1977; Ono \& Comerford, 1977). If one conceives that the IEs influence the shaft such that any component of the "bend" in the angles is perceived to lie toward the shaft, then an illusion, intermediate in magnitude, corresponding to that component will be observed. A slight tilt toward the shaft would cause the concave side of the IEs to face toward the shaft, whereas a slight tilt away from the shaft would cause the concave side of the IEs to face away from the shaft. The reader can get a sense of this from reviewing Figure 7, in which, despite the pictorial depth, the IEs of the right half appear to be bent toward the shaft and those of the left half bent away from the shaft.

\section{GENERAL DISCUSSION}

It has been demonstrated in Experiments 1 and 2 that the Müller-Lyer illusion occurs easily with 3-D figures. Also, it has been shown in Experiments 2 and 3 that the 3-D representation of the patterns may be critical to the understanding of the illusion.

In light of these findings, let us examine the two theories discussed earlier: the constancy scaling theory (Gregory, 1963, 1966, 1968) and the selective spatial frequency filtering theory (Ginsburg, 1984, 1986). Numerous experimenters have already shown that certain predictions that ought to follow from the constancy scaling hypothesis do not hold. For example, Waite and Massaro (1970) reasoned that if a constancy operation were at the root of the illusion, then a rectangle, substituted for the shaft, ought also to show a width distortion in addition to the length distortion. Their studies, however, revealed no such effect. It is possible, however, that constancy scaling occurs only along one dimension and not the other. Some authors (e.g., Day, 1972) have argued that the constancy scaling theory is too specific to be regarded as a general explanation. For example, the variants shown in Figures $2 \mathrm{~b}$ and $2 \mathrm{c}$ both yield the illusion, with some reduction in magnitude, and yet neither of them contains any of the known depth features necessary for the constancy scaling mechanism. The illusion found in these variants shows that the constancy scaling theory is limited in scope because of its intimate link to the perspective depth features. One could, however, argue that the illusion obtained with, for example, the "curves" version of the MüllerLyer is due to mechanisms unrelated to constancy because, after all, the properties of this stimulus are quite different from those of the classical pattern. Furthermore, the reduction in the magnitude of the illusion might also reflect involvement of new mechanisms. What this amounts to is the claim that these variants represent a more or less different illusion.

Such arguments, however, do not hold for the 3-D Müller-Lyer figures studied in Experiments 1 and 2. In terms of the illusion strength, these figures match the classical illusion. Furthermore, in Experiment 2 these figures were shown to be homologous with the classical figure, which suggests the involvement of a common causal mechanism underlying the two types of figures.

Is it possible that constancy scaling might produce the illusion found in the 3-D pattern? Obviously, the dihedral angles of the 3-D figures do not contain perspective in- 
formation that could trigger primary scaling that in turn would compute a distance for the shaft of the longer half greater than the distance for the shaft of the shorter half. Furthermore, the IEs of the "dotted-planes" figure of Experiments 1 and 2 contained no lines that would be considered an important stimulus property for size scaling due to perspective. The presence of the illusion in other figures that do not contain lines was also found by Coren (1970), who used the classical figure made out of dots. In summary, an illusion was observed in the 3-D figure, which was shown to be homologous with the classical figure. Since this 3-D illusion does not lend itself to the constancy scaling interpretation, it is unlikely that constancy scaling is an important contributor to the production of the classical illusion as well.

The three techniques discussed earlier (subjective contours, binocular presentation, and cyclopean presentation) represented attempts to isolate the location along the information-processing pathway at which the Müller-Lyer illusion might occur. In all these studies, the following question was addressed: Is the neural processing at the retina and/or peripheral processes (prior to LGN) caused by a pattern such as the Müller-Lyer necessary for producing the illusion?

The 3-D illusion patterns in Experiments 1 and 2 directly address the involvement of retinal processes. First, it is clear that the retinal neural interactions due to the 3-D patterns are very different from the interactions due to the classical illusion, simply because their 3-D shapes create a very different distribution of stimulation of neural units across the retina. The resulting alteration in the neural interactions ought to have affected the illusion, but an illusion of magnitude comparable to that of the classical pattern was obtained. Furthermore, retinal processes should not be affected by monocular or binocular viewing of the dotted-planes pattern of Experiment 2, yet enormously different illusion magnitudes were obtained in these two conditions. These observations support the view that a retinal image in the shape of the Müller-Lyer figure is not necessary for producing the illusion.

The issue of whether the retinal image of the classical pattern is sufficient for producing the illusion seems to have been neglected. In the cyclopean presentation, for example, once the shape of the illusion pattern is recovered by the comparison of the two images, processes such as selective filtering could become operative and, in principle, cause the illusion. The question of the sufficiency of the retinal image (addressed in Experiment 3) allows the assessment of peripheral processes in addition to processes such as selective spatial filtering. In both conditions of Experiment 3 (planes-parallel/perpendicular), the shape of the retinal image is the same. The selective filtering process, like that proposed by Ginsburg (1984, 1986), should produce identical distortions for the two conditions. The observed illusion for the planesperpendicular condition was, however, half the magnitude of that of the planes-parallel condition. The crucial role played by the orientation of the IEs relative to the shaft in 3-D space suggests that the Müller-Lyer illusion occurs after the 3-D processing.

There are, however, other possibilities that might explain the reduction in the illusion. For example, it is possible that the main illusion occurs primarily due to spatial filtering applied to the image, but that some later 3-D process weakens the effect. This could be a cognitive or judgmental process, or a neural mechanism that alters the output of selective filtering. This of course is speculative, but the plausibility and/or evidence for such a mechanism will determine whether or not selective filtering is indeed a significant factor.

Briefly, the results of Experiment 3 suggest the following. If a selective filtering function were applied to the planes-perpendicular display, the resulting distortions would be identical to the distortion that results when the same function is applied to the planes-parallel display. And yet the magnitude of the illusion obtained by human observers is enormously different for the two displays. Thus, the selective filtering function is "blind" to the 3-D properties of the pattern. The filtering hypothesis may possibly be extended to explain the diminution of the illusion found with the planes-perpendicular display, but only if plausible 3-D processes are discovered.

There is a somewhat different interpretation of the results of the planes-perpendicular condition that may be of interest. If the objects shown in Figure 7 are mentally rotated, we find that they are identical (in 3-D shape), except for a $180^{\circ}$ rotation around the vertical axis (see Shepard \& Metzler, 1971). It is clear, however, that the shapes of the retinal images and the ensuing processes prior to the computation of depth that are yielded by these figures are not identical. The difference between the two projected shapes yielding two different distributions of processes on the surface of the retina might, in principle, produce an illusion, but their identity in 3-D space should oppose any illusion, simply because identical figures should yield processes that are at least in part identical. The finding that the illusion was not produced (assuming for the sake of the argument that the illusion actually observed was entirely due to inaccurate depth perception) suggests that the processes that establish the identity of the two figures in 3-D space are crucial. It would be interesting to find out whether or not performing an explicit mental rotation prior to length judgments would further reduce the illusion, as well as whether or not the illusion is affected when the two objects are used in an apparent motion display in which the "same" object rotates in depth. In another terminology, the findings above mean that the illusion occurs beyond the stage of viewer-centered processing and may fall in the category of object-centered perception (Marr, 1982). Again, the fact that some illusion is produced with the planesperpendicular display is to be expected, since the subjects did not perceive the correct perpendicular orientation of the IEs relative to the shaft.

Some authors, most notably Coren and Girgus (1973), have favored a multimechanism approach. There is general 
agreement regarding the visual information processed at various levels. Is it not then likely that several different levels may simultaneously contribute to the Müller-Lyer illusion? The finding of Coren and Girgus (1978) that a greatly reduced illusion was obtained with the cyclopean version might suggest that peripheral processing is an important contributor in the formation of the illusion. In light of Experiment 3, such a view might imply that the $8.04 \%$ illusion obtained with the planes-perpendicular display is caused by mechanisms that are unaffected by the perpendicular orientation of the IEs relative to the shaft (e.g., retinal interaction and optical blur). On the other hand, the reduction in the illusion magnitude from $16.07 \%$ may be due to the elimination of some of the other cortical mechanisms that are sensitive to orientation. However, as was discussed above, the results of Experiments 1 and 2 cast a further doubt on the strength of the peripheral factors. Moreover, such a view would account for the results fairly well, were it not for the observation that the subjects misperceived the orientation of the IEs. Since it is being argued here that the depth processing mechanism may be located before the illusion-causing mechanism, it follows that any error in the output of the former mechanism will be transmitted to the latter. Presently, experiments in which the orientation of the IEs is veridically perceived are being undertaken to address this issue.

\section{REFERENCES}

Brentano, F. (1892). Über ein optisches Paradoxen. Journal of Psychology, 3, 349-358.

COREN, S. (1970). Lateral inhibition and geometric illusion. Quarterly Journal of Experimental Psychology, 22, 274-278.

Coren, S., \& Girgus, J. S. (1973). Visual spatial illusions: Many explanations. Science, 179, 503-504.

Coren, S., \& Girgus, J. S. (1978). Seeing is deceiving: The psychology of visual illusions. Hillsdale, NJ: Erlbaum.

DAY, R. H. (1972). Visual spatial illusions: A general explanation. Science, 175, 1335-1340.

Delboeuf, J. L. R. (1892). Sur une nouvelle illusion d'optique. Academie Royale des Sciences, de Lettres et des Beaux Arts de Belgique Bulletins, 24, 545-558.

Delucia, P., Hochberg, J. (1985). Illusions in the real world and in the mind's eye. Proceedings of the Eastem Psychological Association, 56, 38. (Abstract)

DeLUCIA, P., \& HOCHBERG, J. (1986). Real-world geometrical illusions: Theoretical and practical implications. Proceedings of the Eastern Psychological Association, 57, 62. (Abstract)

DeVAloIs, R. L., \& DeVAloIs, K. K. (1988). Spatial vision. New York: Oxford University Press.

GrBson, J. J. (1966). The senses considered as perceptual systems. Boston: Houghton Mifflin.

GinsBuRG, A. P. (1984). Visual form perception based on biological filtering. In L. Spillman \& B. R. Wooten (Eds.), Sensory experience, adaptation and perception (pp. 53-72). Hillsdale, NJ: Erlbaum.

GinsBURG, A. P. (1986). Spatial filtering and visual form perception. In K. R. Boff, L. Kaufman, \& J. P. Thomas (Eds.), Handbook of perception and human performance (Vol. 2, pp. 34-1 to 34-41). New York: Wiley.

GoGEL, W. (1977). The metric of visual space. In W. Epstein (Ed.), Stability and constancy in visual perception: Mechanisms and processes (pp. 129-181). New York: Wiley.

Goldstein, M. B., \& Weintraub, D. J. (1972). The parallel-less Poggendorff: Virtual contours put the illusion down but not out. Perception \& Psychophysics, 11, 353-355.

GrEGORY, R. L. (1963). Distortion of visual space as inappropriate constancy scaling. Nature, 199, 678-680.

Gregory, R. L. (1966). Eye and brain. New York: World University Library.

Gregory, R. L. (1968). Visual illusions. Scientific American, 219, 66-76.

JULEsz, B. (1971). Foundations of cyclopean perception. Chicago: University of Chicago Press.

Kanizsa, G. (1974). Contours with gradients or cognitive contours? Italian Joumal of Psychology, 1, 93-112.

LEwIs, E. O. (1909). Confluxion and contrast effects in the MüllerLyer illusion. British Joumal of Psychology, 3, 21-41.

MarR, D. (1982). Vision. San Francisco: W. H. Freeman.

MülLER-LyER, F. C. (1889). Optische Urteilstäuschungen. DuboisReymonds Archive für Anatomie und Physiologie (Suppl.), 263-270.

ONo, H., \& Comerford, J. (1977). Stereoscopic depth constancy. In W. Epstein (Ed.), Stability and constancy in visual perception: Mechanisms and processes (pp. 91-128). New York: Wiley.

PAPERT, S. (1961). Centrally produced geometrical illusions. Nature, $191,733$.

SCHILler, P., \& WeINER, M. (1962). Binocular and stereoscopic viewing of geometric illusions. Perceptual \& Motor Skills, 13, 739-747.

SHEPARD, R. N., METZler, J. (1971). Mental rotation of threedimensional objects. Science, 171, 701-703.

WaIte, H., \& MASSARO, D. W. (1970). A test of Gregory's constancyscaling explanation of the Müller-Lyer illusion. Nature, 227, 733-734.

(Manuscript received March 7, 1988;

revision accepted for publication November 15, 1990.) 